\title{
Administração de medicamentos via sonda enteral no pós-operatório gastrointestinal
}

\author{
Drug administration through enteral tubes after gastrointestinal surgery \\ Administración de medicamentos por sonda enteral en el posoperatorio gastrointestinal
}

Recebido: 11/04/2021 | Revisado: 18/04/2021 | Aceito: 23/04/2021 | Publicado: 09/05/2021

\author{
Sandra Regina dos Santos \\ ORCID: https://orcid.org/0000-0001-5010-5933 \\ Universidade Federal de Minas Gerais, Brasil \\ E-mail: sandrasantos0105@gmail.com \\ Raquel Linhares Bello de Araújo \\ ORCID: https://orcid.org/0000-0002-5110-5203 \\ Universidade Federal de Minas Gerais, Brasil \\ E-mail: raquel@bromatologiaufmg.com.br \\ Cristiane de Paula Rezende \\ ORCID: https://orcid.org/0000-0001-7457-4187 \\ Universidade Federal de Minas Gerais, Brasil \\ E-mail: cris7paula@gmail.com \\ Mariana Martins Gonzaga do Nascimento \\ ORCID: https://orcid.org/0000-0003-2183-4365 \\ Universidade Federal de Minas Gerais, Brasil \\ E-mail: marianamgn@yahoo.com.br
}

\begin{abstract}
Resumo
Pacientes submetidos a operações do trato gastrointestinal são complexos e frequentemente recebem medicamentos via sondas enterais (SE). O objetivo desse estudo foi analisar o perfil e fatores associados às limitações da administração via SE de medicamentos prescritos para pacientes submetidos a operações do trato gastrointestinal e parede abdominal. Para tal, analisou-se todas as prescrições eletrônicas de pacientes sob uso de nutrição enteral (NE) em pós-operatório em um hospital universitário geral (01/05 a 05/12/2017). Medicamentos prescritos para administração via SE foram identificados e realizou-se uma revisão da literatura acerca de limitações para sua administração por esta via. Determinou-se a prevalência dessas limitações e fatores associados por meio de análises uni (qui-quadrado de Pearson) e multivariada (regressão logística - nível de significância estatística de 5\%). Foram analisadas 341 prescrições de 40 pacientes. Identificou-se 725 medicamentos prescritos via SE (44 medicamentos diferentes). Na revisão, recuperou-se 33 artigos para construção de uma base de dados, que permitiu a identificação de 188 limitações quanto à administração via SE entre os medicamentos prescritos, e uma prevalência de pelo menos uma limitação de 57,5\% (n=23), que mostrou-se associada positivamente à idade igual ou superior a 60 anos (OR 4,67; IC95\% 1,07-20,40). Devido à escassez de referências voltadas para administração de medicamentos via SE, esse estudo configura uma importante ferramenta para o cuidado farmacoterapêutico ao paciente sob uso de NE. O número de limitações identificado foi reduzido, mas sua associação com idade avançada e a gravidade da população estudada reforçam a importância da priorização de seu manejo.

Palavras-chave: Preparações farmacêuticas; Nutrição enteral; Interações medicamentosas; Procedimentos cirúrgicos operatórios; Cuidados pós-operatórios.
\end{abstract}

\begin{abstract}
Patients undergoing gastrointestinal tract operations are complex and often receive medications via enteral tubes (ET). The aim of this study was to analyze the profile of the limitations of administration via SE of medications prescribed to patients undergoing operations on the gastrointestinal tract and abdominal wall and associated factors. To this end, all electronic prescriptions of patients using enteral nutrition $(\mathrm{EN})$ and in the postoperative period at a general university hospital were analyzed (01/05 to 12/05/2017). Medicines prescribed for administration via ET were identified and a literature review about limitations for its administration in this type of tube. The prevalence of these limitations and associated factors was determined through uni (Pearson's chi-square) and multivariate (logistic regression - 5\% statistical significance level) analyzes. A total of 341 prescriptions from 40 patients were analyzed. A total of 725 drugs prescribed via ET (44 different drugs) were identified. In the review, 33 articles were retrieved for the construction of a database, which allowed the identification of 188 limitations regarding drug administration via ET. The prevalence of at least one limitation regarding drug administration via ET among the patients evaluated was $57.5 \%(\mathrm{n}=23)$, and it was positively associated with age equal to or greater than 60 years (OR 4.67; 95\%CI 1.07 20.40). Due to the scarcity of references for medication administration via ET, this study is an important tool for pharmacotherapeutic care for patients using EN. The number of limitations identified was low, but its association with advanced age and the severity of the studied population reinforce the importance of prioritizing its management.
\end{abstract}


Keywords: Pharmaceutical preparations; Enteral nutrition; Drug interactions; Surgical procedures operative; Postoperative care.

\begin{abstract}
Resumen
Los pacientes que se someten a operaciones del tracto gastrointestinal son complejos y, frecuentemente, reciben medicamentos a través de sondas enterales (SE). El objetivo de este estudio fue analizar el perfil de las limitaciones de la administración vía SE de medicamentos prescritos a pacientes sometidos a operaciones en el tracto gastrointestinal y pared abdominal y factores asociados. Para esto, se analizaron todas las prescripciones electrónicas de pacientes en nutrición enteral (NE) y en el postoperatorio de un hospital universitario general (01/05 al 12/05/2017). Se identificaron los medicamentos prescritos para su administración vía SE y se realizó una revisión de la literatura sobre las limitaciones para su administración en este tipo de sonda. La prevalencia de estas limitaciones y factores asociados se determinó mediante análisis univariante (chi-cuadrado de Pearson) y multivariante (regresión logística - nivel de significación estadística del 5\%). Se analizaron un total de 341 prescripciones de 40 pacientes. Se identifico un total de 725 medicamentos prescriptos a través de SE (44 medicamentos diferentes). En la revisión, se recuperaron 33 artículos para la construcción de una base de datos, lo que permitió identificar 188 limitaciones en cuanto a la administración de fármacos vía SE. La prevalencia de al menos una limitación respecto a la administración de fármacos vía SE entre los pacientes evaluados fue del 57,5\% $(\mathrm{n}=23)$, y se asoció positivamente con la edad igual o superior a 60 años (OR 4,67; IC 95\% 1,07-20,40). Debido a la escasez de referencias para la administración de medicamentos a través de SE, este estudio es una herramienta importante para la atención farmacoterapéutica de los pacientes que utilizan NE. El número de limitaciones identificadas fue bajo, pero su asociación con la edad avanzada y la gravedad de la población estudiada refuerzan la importancia de priorizar su manejo.

Palabras clave: Preparaciones farmacéuticas; Nutrición enteral; Interacciones farmacológicas; Procedimientos quirúrgicos operativos; Cuidados posoperatorios.
\end{abstract}

\title{
1. Introdução
}

Pacientes submetidos a operações do trato gastrointestinal são complexos e apresentam alterações metabólicas relacionadas ao estresse causado pela operação (Huang, Sun, Huang, \& Shen, 2015). Além disso, esses pacientes frequentemente apresentam desnutrição, que, por sua vez, está associada a danos como comprometimento da sua composição corporal, função muscular e da cicatrização de anastomoses (Ahn et al., 2011; Huang et al., 2015; Lewis, Andersen, \& Thomas, 2009; Zhao, Wu, Zhao, Liu, \& Dai, 2016).

Nesse cenário, estudos apontam para a relevância do uso de NE para a recuperação do paciente no pós-operatório gastrointestinal, proporcionando-lhe melhor cicatrização, manutenção funcional e estrutural intestinal, menor tempo de internação hospitalar e complicações sépticas e não sépticas (Ahn et al., 2011; Huang et al., 2015; Lewis et al., 2009; Wheble, Knight, \& Khan, 2012; Zhuang et al., 2013). Portanto, o uso de NE entre esses pacientes tem sido cada vez mais recomendado e usual, trazendo consigo o desafio do uso concomitante de NE e medicamentos via sondas ou ostomias (Boullata, 2009; Phillips \& Endacott, 2011).

A administração de medicamentos via sondas e ostomias envolve riscos consideráveis, podendo aumentar a morbidade e mortalidade (Phillips \& Nay, 2008). Algumas das consequências da administração de medicamentos via sonda incluem: alteração da forma farmacêutica original (trituração de comprimidos); oclusão da sonda e possível necessidade de sua repassagem; desconhecimento da localização da ponta distal da sonda, ou deslocamento dessa, e consequente liberação do medicamento em local diferente do sítio de absorção indicado; e interação do fármaco com os componentes da NE ou com a própria sonda (DRUG-REAX®, 2018; Kozeniecki \& Fritzshall, 2015; Krajewski \& Butterfoss, 2011; Miller, McClave, Kiraly, Martindale, \& Benns, 2014; Phillips \& Endacott, 2011; Reis et al., 2014; Silva \& Lisboa, 2011; Zhuang et al., 2013).

Apesar da ciência dos desafios da administração de medicamentos e NE pela mesma via, ainda não há uma base de dados específica para consulta sobre a temática. Além disso, essa área é carente de estudos primários recentes, com método robusto e que abranjam um número expressivo de medicamentos (Nascimento \& Ribeiro, 2010; Silva, Cava, Pedroso, \& Futuro, 2011). Dessa forma, os profissionais acabam precisando buscar conhecimento quanto às limitações da administração de medicamentos via sonda em uma literatura que ainda não está devidamente consolidada (Nascimento \& Ribeiro, 2010; 
Silva et al., 2011). A escassez é ainda mais pronunciada quando deseja-se identificar informações específicas para a avaliação de pacientes cirúrgicos, que apresentam quadros clínicos de usual fragilidade e de difícil manejo. O objetivo desse estudo foi, portanto, analisar o perfil e fatores associados às limitações da administração de medicamentos via sonda nasoenteral entre pacientes submetidos a operações do trato gastrointestinal e de parede abdominal durante o período pós-operatório.

\section{Metodologia}

Trata-se de um estudo misto com um componente transversal e um componente de revisão da literatura integrativa. Dividido em 3 etapas, foi realizado em um hospital público, geral, universitário e integrado ao Sistema Único de Saúde (SUS) brasileiro, localizado em Belo Horizonte - Minas Gerais. A enfermaria onde foi desenvolvido conta com 28 leitos clínicocirúrgicos e oferece tratamentos das enfermidades do aparelho digestivo.

\section{Etapa I - Análise de prescrições}

Analisou-se todas as prescrições eletrônicas elaboradas no período (definido por conveniência) de 01 de maio de 2017 a 05 de dezembro de 2017 para a população de pacientes internados na referida enfermaria (contabilizando 40 pacientes e 341 prescrições). Foram utilizados os seguintes critérios de inclusão: (1) paciente em pós-operatório; (2) sob uso de nutrição enteral (qualquer fórmula); (3) e em uso de pelo menos um medicamento via sonda.

Das prescrições e prontuários dos pacientes, foram coletadas informações sobre: seu perfil demográfico; número e tipo de doenças; hábitos de vida; diagnóstico principal; tipo e porte da operação realizada; especialidade envolvida na operação; presença de desnutrição grave; perfil de utilização de NE; e medicamentos prescritos para serem administrados via sondas. A análise descritiva desses dados foi realizada mediante determinação das frequências absolutas e relativas das variáveis qualitativas; e média, desvio padrão, mínimo e máximo das variáveis quantitativas.

\section{Etapa II - Revisão de literatura}

A partir da lista de medicamentos prescritos para serem administrados via sondas identificados na primeira etapa, procedeu-se uma revisão da literatura sobre administração desses via sonda. Para isso, foram consultadas as bases de dados PubMed, da biblioteca Medline (Medical Literature Analysis and Retrieval System Online) e Lilacs (Literatura LatinoAmericana e do Caribe em Ciências da Saúde). Foi utilizada a seguinte estratégia de combinação de descritores e termos livres: ("drug" OR "pharmaceutical preparations") AND ("enteral nutrition" OR "nasogastric tube" OR "gastric tube" OR "enteral tube" OR "enteral feed" OR "orogastric tube" OR "nasogastric tube" OR "nasogastric feeding" OR "gastric feeding" OR "enteral feed" OR "nasoenteric tube" OR "oroenteric tube" OR "feed tube" OR "enteric feeding" OR "intubation, gastrointestinal").

Aplicou-se um limite de idiomas nas bases de dados consultadas, sendo recuperados apenas artigos nos idiomas inglês, português ou espanhol. A seleção dos artigos recuperados foi então composta por duas etapas: inicialmente pelo título e resumo do artigo; e, por último, pela leitura do texto na íntegra. Os artigos foram selecionados por dois pesquisadores independentes e, em caso de desacordo entre estes, a inclusão do artigo para análise na íntegra foi definida por um terceiro pesquisador. Foram incluídos artigos que continham informações sobre o preparo e administração via sonda de pelo menos um dos medicamentos prescritos identificados na Etapa I. Foram excluídos estudos realizados em animais.

Além disso, informações sobre os medicamentos identificados na Etapa I foram buscadas na fonte terciária Handbook of Drug Administration via Enteral Feeding Tubes (Boullata \& Armenti, 2010), e na base de dados Drug-Reax® da Micromedex® (DRUG-REAX®, 2018). Informações sobre o conteúdo de sorbitol de soluções e suspensões orais foi consultado em bulas disponibilizadas pelos fabricantes dos medicamentos. 
A partir da revisão de literatura, foi construído o compilado de dados com características sobre técnicas de preparo e administração mais adequadas, possibilidade de ocorrência de interação medicamento-nutrição enteral, osmolaridade e teor de sorbitol (no caso de suspensões e soluções orais) e outras recomendações pertinentes relacionadas à administração via sonda.

\section{Etapa III - Limitações quanto à administração em sonda enteral}

Com base no compilado de dados elaborado a partir da etapa II, foram identificadas as limitações quanto à administração em sonda enteral nas prescrições analisadas. Considerou-se que um medicamento apresentava limitações quanto à administração em sonda enteral, quando foi identificada no compilado de dados informações sobre: (1) interações entre o medicamento e NE; e (2) necessidade de forma de preparo e/ou administração diferenciada do medicamento; ou (3) ambas as limitações citadas anteriormente.

Foram determinadas as frequências absolutas e relativas das limitações quanto à administração em sonda enteral de acordo com o medicamento. Também se determinou a prevalência de pelo menos uma limitação quanto à administração em sonda enteral entre os pacientes incluídos no estudo, determinada como variável dependente. Para determinar os fatores associados a essa variável dependente, foram realizadas análises univariadas e multivariada com as seguintes variáveis independentes, que foram dicotomizadas conforme mediana ou relevância clínica:

- Demográficas: sexo, idade (18 a 59 anos versus 60 anos ou mais, considerado ponto de corte para idosos em países em desenvolvimento);

- $\quad$ Número de medicamentos utilizados via sonda na primeira prescrição (1 medicamento versus 2 ou mais)

- $\quad$ Localização da ponta distal da sonda (estômago versus outros);

- Diagnóstico das doenças mais frequentes na população estudada: neoplasia (sim versus não), hipertensão (sim versus não), diabetes mellitus (sim versus não);

- $\quad$ Tipo de operação mais frequentes: operação de câncer de cabeça e pescoço (sim versus não); operação de esôfago, estômago e duodeno (sim versus não).

Análises univariadas foram realizadas utilizando-se teste de qui-quadrado de Pearson ou teste exato de Fisher quando o valor esperado de uma ou mais células foi igual ou inferior a cinco. Tendo em vista a ausência na literatura de investigação prévia de fatores associados no cenário proposto no presente estudo, optou-se por adotar um modelo multivariado parcimonioso, sendo que todas as variáveis foram incluídas no modelo final, independentemente de qualquer critério estatístico. O modelo multivariado foi calculado por meio de regressão logística stepwise, e a qualidade de seu ajuste foi avaliada pelo teste Hosmer-Lemeshow. Análises univariadas e multivariada foram baseadas na medida de Odds Ratio (OR) e no seu respectivo intervalo de confiança de 95\% (IC95\%), estimados por regressão logística. O nível de significância de 5\% foi o critério adotado para identificar características independentemente associadas à variável dependente.

Informações coletadas foram digitadas no programa Excel® e posteriormente transferidas e organizadas em banco de dados do software Stata® versão 12 (StataCorp. College Station, Estados Unidos). Para todas as análises estatísticas foi utilizado esse último software.

\section{Aspectos éticos}

A execução deste estudo foi aprovada pelo Comitê de Ética em Pesquisa da UFMG (COEP), sob registro CAAE72758617.3.0000.5149 e foi desenvolvido respeitando todos os princípios éticos constantes na Resolução n ${ }^{\circ} 466$, de 12 de dezembro de 2012, sobre pesquisa envolvendo seres humanos. 


\section{Resultados}

Foram analisados dados referentes a 341 prescrições de 40 pacientes (média de 8,52 prescrições por paciente). A maioria dos pacientes era do sexo masculino $(65,0 \% ; n=26)$ e identificou-se uma média de idade de $60,5 \pm 13,9$ anos (mínima: 22; máxima: 81), revelando alto número de idosos (indivíduos com 60 anos ou mais $=26$; 65\%).

A média de problemas de saúde documentados nos prontuários por paciente além do diagnóstico principal foi de

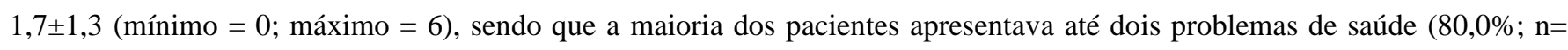
32). Vinte e oito (70,0\%) desses pacientes foram submetidos a operações de grande porte. Em 17 (42,5\%) pacientes, a ponta distal da sonda estava localizada no estômago; em outros 3 pacientes $(7,5 \%)$ localizava-se no jejuno; e para os demais $(n=20$; $50 \%$ ), essa localização não foi documentada em prontuário.

As doenças mais frequentes na população estudada foram: neoplasia $(60,0 \% ; n=24)$, hipertensão arterial sistêmica $(37,5 \% ; \mathrm{n}=15)$, e diabetes mellitus $(15,0 \% ; \mathrm{n}=6)$. O diagnóstico principal mais frequente foi neoplasia de estômago (20,0\%; $\mathrm{n}=8)$, seguido por neoplasia de esôfago $(15,0 \% ; \mathrm{n}=6)$ e de laringe $(15,0 \% ; \mathrm{n}=6)$. Poucos pacientes eram etilistas $(10,0 \% ; \mathrm{n}=4)$ ou tabagistas $(12,5 \%$; $=5)$, mas parte considerável apresentou desnutrição grave $(42,5 \%$; n=17).

Os pacientes incluídos também foram classificados de acordo com o grupo de especialidade cirúrgica envolvida na sua operação. A maioria dos pacientes foi atribuída à especialidade de "Esôfago, Estômago e Duodeno" (n=22; 55,0\%), seguida pelas especialidades "Câncer de Cabeça e Pescoço" (n=14, 35,0\%), "Coloproctologia" (n=2; 5,0\%), "Fígado e Vias Biliares" ( $n=1 ; 2,5 \%)$ e "Parede Abdominal" ( $n=1 ; 2,5 \%)$.

Foram identificados 725 itens de medicamentos prescritos para serem administrados pela sonda enteral (média de

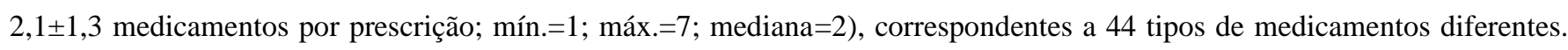
Desses medicamentos, $528(72,8 \%)$ apresentavam-se sob forma farmacêutica sólida e os demais, formas farmacêuticas líquidas.

Foi realizada a revisão de literatura referente aos 44 medicamentos identificados na Etapa I para a elaboração de um compilado de dados sobre a administração de medicamentos via sondas enterais. A estratégia de busca para a revisão retornou um total de 1.493 artigos. Na Figura 1, é apresentado o fluxograma de seleção de artigos científicos de acordo com as etapas propostas nos métodos e critérios de exclusão adotados. 
Figura 1. Fluxograma de seleção de artigos. Belo Horizonte - MG. 2018.

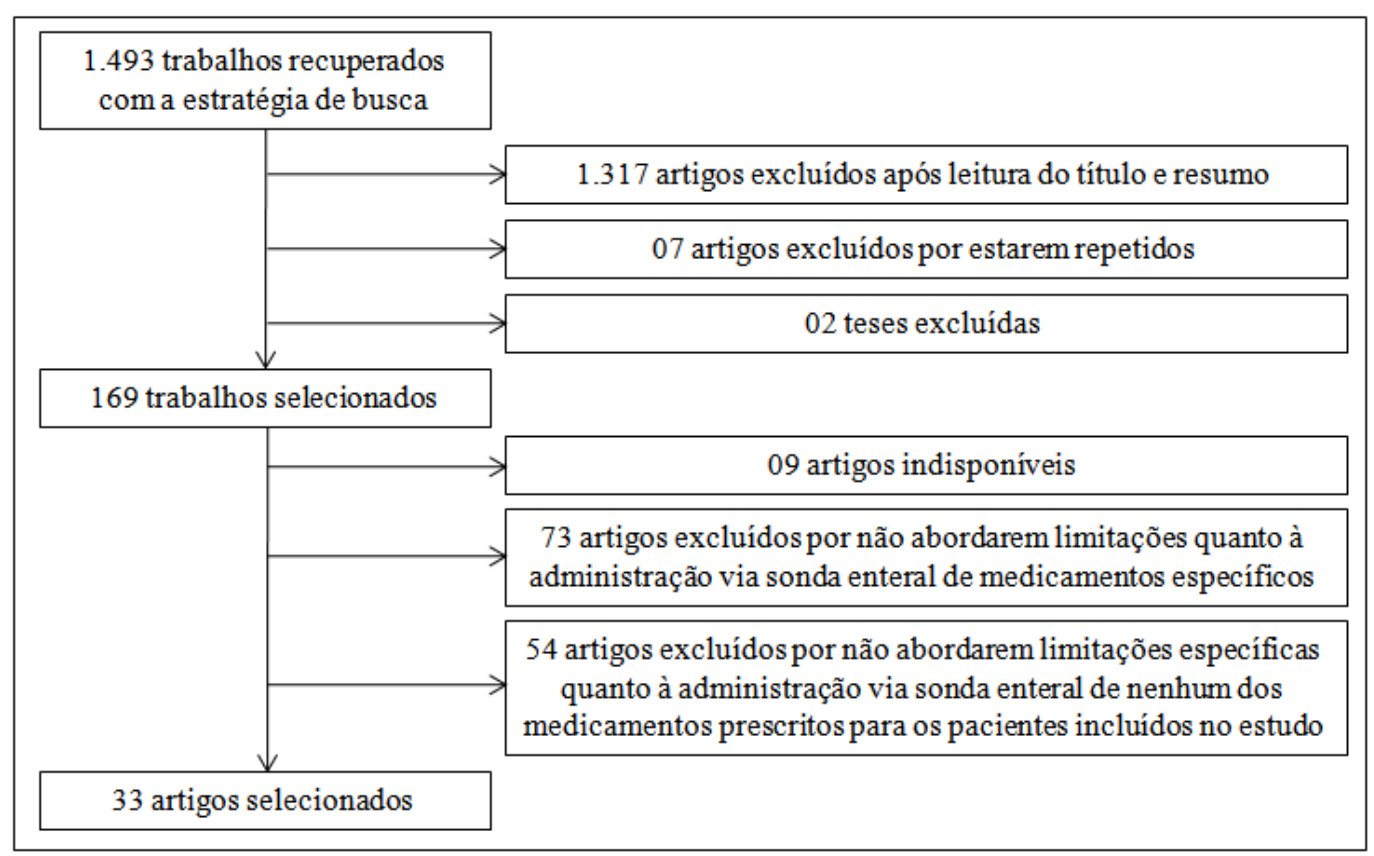

Fonte: Autores (2018).

Após análise, 33 artigos atenderam os critérios de inclusão e as respectivas informações foram utilizadas para o desenvolvimento da Tabela 1.

Tabela 1. Orientações sobre técnica e/ou limitações no preparo e administração dos medicamentos prescritos para administração em sonda no hospital estudado. Belo Horizonte - MG. 2019.

\begin{tabular}{|c|c|}
\hline $\begin{array}{l}\text { Medicamento/ } \\
\text { fármaco (Forma } \\
\text { farmacêutica) }\end{array}$ & Interações/ Preparos/ Incompatibilidades/ Recomendações \\
\hline
\end{tabular}

Forma farmacêutica: forma líquida preferível - solução oral ou xarope (Elke Joos, Verbeke, et al., 2016; Mink, Muroi, Seule, Bjeljac, \& Keller, 2011; L. D. Silva \& Lisboa, 2011); opção de uso de comprimido revestido não triturável (E. Joos et al., 2015; Elke Joos, Mehuys, Remon, Van Winckel, \& Boussery, 2016; Elke Joos, Verbeke, et al., 2016; L. D. Silva \& Lisboa, 2011).

Interações potenciais: sem limitação de absorção documentada para sonda gástrica ou duodenal (Mink et al., 2011); possível limitação na absorção do fármaco quando administrado pela via jejunal (Jory, Shankar, Oak, Oates, \& Ácido valproico Wilcock, 2017).

(Solução oral) Forma de preparo e administração: diluir forma líquida em volume equivalente ou superior de água (E. Joos et al., 2015); não triturar comprimidos com revestimento (destruição do revestimento provoca irritação da mucosa e a inativação ou liberação rápida do fármaco) (E. Joos et al., 2015; Elke Joos, Mehuys, et al., 2016; Elke Joos, Verbeke, et al., 2016; L. D. Silva \& Lisboa, 2011).

Informações adicionais: conteúdo de sorbitol do xarope (fabricante: Abbott - medicamento de referência no Brasil) $=0,15 \mathrm{~g} / \mathrm{mL}$ (Boullata \& Armenti, 2010).

Forma farmacêutica: comprimido pode ser triturado (Decloedt \& Maartens, 2009).

Amiodarona (Comprimido)
Interações potenciais: fármaco pode se adsorver a sondas de policloreto de vinila (Decloedt \& Maartens, 2009); a biodisponibilidade pode aumentar com administração concomitante de dieta hiperlipídica (Tisdale et al., 2007); concentrações séricas do fármaco mostraram-se significativamente menores e até indetectáveis em pacientes que receberam o medicamento via sonda nasogástrica, quando comparados àqueles que receberam por via oral (Tisdale et al., 2007). 


\title{
Medicamento/ \\ fármaco (Forma \\ farmacêutica) \\ Interações/ Preparos/ Incompatibilidades/ Recomendações
}

Forma de preparo e administração: diluir o comprimido triturado e lavar a sonda com água, cloreto de sódio ou dextrose para diminuir a adsorção (Decloedt \& Maartens, 2009).

Informações adicionais: pode causar náusea (o que pode ser evitado diminuindo-se a dose diária durante a fase inicial) e alteração do paladar (Boullata \& Armenti, 2010).

Forma farmacêutica: comprimido pode ser triturado (Elke Joos, Verbeke, et al., 2016).

\begin{abstract}
Amoxicilina + Forma de preparo e administração: requer uso de equipamento de proteção (máscara e luvas) durante a trituração clavulanato (Pó (Elke Joos, Verbeke, et al., 2016); agitar a suspensão antes do uso para evitar variabilidade nas doses administradas para suspensão (E. Joos et al., 2015).
\end{abstract}

oral)

Informações adicionais: sem sorbitol na formulação (fabricantes: Apothecon, Biocraft, Lederle, SKB e WA) (Boullata \& Armenti, 2010).

\begin{tabular}{cl}
$\begin{array}{c}\text { Anlodipino } \\
\text { (Comprimido) }\end{array}$ & Forma farmacêutica: comprimidos dispersam em água (Kelly, Wright, \& Wood, 2011). \\
\hline $\begin{array}{c}\text { Atenolol } \\
(\text { Comprimido) }\end{array}$ & $\begin{array}{l}\text { Interações potenciais: biodisponibilidade significativamente reduzida quando comprimidos triturados são } \\
\text { administrados via sonda nasoenteral no pós operatório, em comparação com comprimidos intactos administrados no } \\
\text { pré-operatório (Decloedt \& Maartens, 2009). }\end{array}$ \\
\hline
\end{tabular}

Bicarbonato de sódio (Pó para reconstituição para solução oral)

Informações adicionais: estudos disponíveis não avaliam uso de bicarbonato de sódio via sonda isoladamente (Bourgault, Ipe, Weaver, Swartz, \& J. O’Dea, 2007; Johnson, Cober, \& Ludwig, 2007).

Forma farmacêutica: se possível, utilizar formulação líquida (Renovato, de Carvalho, \& Rocha, 2010); comprimido pode ser triturado (Renovato et al., 2010).

Captopril (Comprimido)

Interações potenciais: administração conjunta de captopril e nutrição enteral reduz absorção entre 30 e $50 \%$ (Renovato et al., 2010); captopril forma complexos estáveis com ferro (Boullata \& Armenti, 2010).

Forma de preparo e administração: comprimido pode ser triturado, dissolvido e administrado imediatamente (Renovato et al., 2010); recomenda-se interromper a nutrição 30 minutos antes da administração e iniciar somente 30 minutos depois (Renovato et al., 2010; M. F. B. da Silva, Brito, \& Guaraldo, 2016).

Forma farmacêutica: se possível, utilizar formulação líquida (E. Joos et al., 2015; Renovato et al., 2010).

Interações potenciais: fármaco pode se adsorver a sondas de policloreto de vinila (Decloedt \& Maartens, 2009; Wilson \& Best, 2011).

Forma de preparo e administração: comprimido deve ser dissolvido em água e administrado imediatamente (Renovato et al., 2010); deve-se agitar a suspensão antes do uso (E. Joos et al., 2015; Elke Joos, Verbeke, et al., Carbamazepina 2016); diluir as formulações e lavar a sonda com água, cloreto de sódio ou dextrose para diminuir a adsorção e (Comprimido) oclusão (Decloedt \& Maartens, 2009; E. Joos et al., 2015; Wilson \& Best, 2011); recomendado interromper a nutrição enteral por pelo menos 30 minutos antes e após a administração (Boullata, 2009); não triturar comprimidos de liberação prolongada (ocasiona liberação imediata da dose total) (E. Joos et al., 2015; Elke Joos, Mehuys, et al., 2016; Elke Joos, Verbeke, et al., 2016).

Outras informações: duodeno é via preferencial de administração para esse fármaco que pode ser inativado em meio ácido (Heldt \& Loss, 2013).

Interações potenciais: administração no jejuno pode aumentar absorção e efeitos sistêmicos de betabloqueadores

Carvedilol (Kappelle, Siersema, Bogte, \& Vleggaar, 2016).

(Comprimido)

Outras informações: pode causar náuseas, vômitos, diarreia ou constipação (Boullata \& Armenti, 2010).

Ciprofloxacino Forma farmacêutica: deve-se preferir a administração da forma endovenosa, a menos que seja possível o (Comprimido) monitoramento da concentração plasmática do ciprofloxacino (L. D. da Silva, Schutz, Praça, \& Santos, 2010). 


\begin{tabular}{|c|c|}
\hline $\begin{array}{l}\text { Medicamento/ } \\
\text { fármaco (Forma } \\
\text { farmacêutica) }\end{array}$ & Interações/ Preparos/ Incompatibilidades/ Recomendações \\
\hline & $\begin{array}{l}\text { Interações potenciais: forma quelato insolúvel com cátions metálicos da nutrição prejudicando a absorção (ex.: } \\
\text { alumínio, cálcio, magnésio, ferro, cobre e zinco) (Boullata \& Armenti, 2010; Kurien, Penny, \& Sanders, 2015; V. G. } \\
\text { do O. Reis, Candido, Jesus, \& Mendes-Netto, 2010; L. D. da Silva et al., 2010); concentração sérica variável quando } \\
\text { administrado via sonda quando comparado à administração via endovenosa (Boullata \& Armenti, 2010; L. D. da } \\
\text { Silva et al., 2010); absorção é maior quando administrado por sonda nasoduodenal do que por sonda nasogástrica } \\
\text { (fármaco instável no ambiente gástrico) (Heldt \& Loss, 2013; Lubart et al., 2013); Ensure® (produto alimentar } \\
\text { entérico) diminui absorção quando comparado à água (Boullata \& Armenti, 2010). }\end{array}$ \\
\hline
\end{tabular}

Forma de preparo e administração: interromper a NE antes e após a administração (L. D. da Silva et al., 2010), sendo recomendada interrupção entre 1 e 2 horas antes e 2 a 4 horas após (Boullata \& Armenti, 2010; V. G. do O. Reis et al., 2010);lavar a sonda(L. D. da Silva et al., 2010); não triturar comprimidos com revestimento entérico (L. D. da Silva et al., 2010); deve-se agitar a forma líquida antes do uso (E. Joos et al., 2015).

Outras informações: duodeno é via preferencial para esse fármaco que pode ser inativado em meio ácido (Boullata \& Armenti, 2010; Heldt \& Loss, 2013); administração jejunal resulta em menor concentração sérica do que oral (Boullata \& Armenti, 2010); usar antimicrobiano diferente, com cobertura adequada para a infecção, seria opção para evitar a interação (Boullata \& Armenti, 2010).

Cloreto de potássio (Solução injetável e solução oral)

Forma farmacêutica: solução não oclui a sonda (Nicole M. Phillips \& Nay, 2008).

Outras informações: dividir doses de produtos hipertônicos, hiperosmolares ou irritantes, como o cloreto de potássio, em duas a quatro doses menores administradas com tempo de pelo menos 1 hora entre si quando isso não alterar a efetividade (Boullata \& Armenti, 2010).

Forma farmacêutica: uso de forma farmacêutica líquida pode resultar em maior absorção do fármaco (Boullata \& Armenti, 2010; Kelly et al., 2011); comprimidos se dispersam em água (Kelly et al., 2011); medicamento compatível com sonda enteral (Lonergan, Broderick, Coughlan, Collins, \& Neill, 2009).

Interações potenciais: administração concomitante com dietas ricas em fibras pode diminuir a absorção da digoxina (Boullata \& Armenti, 2010).

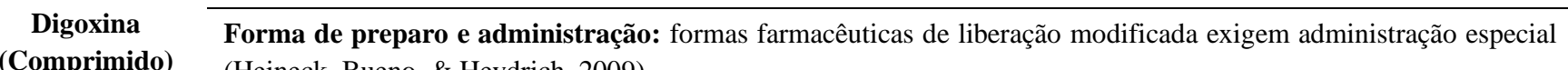
(Comprimido) (Heineck, Bueno, \& Heydrich, 2009).

Outras informações: pode produzir náuseas, vômitos, diarreia, anorexia, ou alteração no paladar ou olfato (Boullata \& Armenti, 2010); esvaziamento gástrico lento aumenta dissolução e diminui absorção (lábil a ácidos) (Boullata \& Armenti, 2010); absorção variável quando administrada no duodeno, sobretudo no jejuno (tempo para dissolução é reduzido) (Boullata \& Armenti, 2010); realizar monitorização terapêutica plasmática do fármaco dentro de alguns dias, sempre que a dose for alterada ou ocorrer mudança de via de administração (Boullata \& Armenti, 2010).

Forma farmacêutica: comprimidos dispersam em água (Kelly et al., 2011).

\section{Espironolactona \\ (Comprimido)}

Outras informações: esvaziamento gástrico lento aumenta dissolução (espironolactona é relativamente insolúvel) (Boullata \& Armenti, 2010).

Forma farmacêutica: forma líquida disponível (Kelly et al., 2011); comprimidos dispersam em água (Kelly et al.,

Furosemida 2011).

(Comprimido) Outras informações: administrar com estômago vazio (aumento da absorção em jejum) (Boullata \& Armenti, 2010); biodisponibilidade reduzida entre 16 e $45 \%$ quando ingerida com alimentos (Boullata \& Armenti, 2010).

Interações potenciais: administração concomitante com a nutrição enteral leva à precipitação da lactulose e redução da absorção de nutrientes (V. G. do O. Reis et al., 2010).

Lactulose (Solução oral)

Forma de preparo e administração: devido à viscosidade, dissolver ou diluir com grande volume de água para evitar oclusão da sonda (Elke Joos, Mehuys, et al., 2016).

Outras informações: frequência de diarreia sem relação com duração do uso do medicamento (Jack, Coyer, Courtney, \& Venkatesh, 2010).

Levotiroxina Forma farmacêutica: comprimido pode ser triturado (Elke Joos, Verbeke, et al., 2016). 


\begin{tabular}{c}
\hline Medicamento/ \\
fármaco (Forma \\
farmacêutica) \\
\hline (Comprimido)
\end{tabular}

Interações potenciais: fármaco se liga diretamente à nutrição enteral, que, por sua vez, é adsorvida à superfície da sonda, podendo diminuir a biodisponibilidade da Levotiroxina (Manessis, Lascher, Bukberg, Darmody, \& Y, 2008); absorção reduzida quando administrada juntamente com alimentos ou NE (Boullata \& Armenti, 2010; DRUGREAX®, 2018; Guenter \& Boullata, 2013; Kurien et al., 2015; Manessis et al., 2008); interações com antiácidos, sulfato de ferro, dietas ricas em fibras e cálcio demonstraram diminuir a absorção da Levotiroxina (Boullata \& Armenti, 2010).

Forma de preparo e administração: requer uso de equipamento de proteção (máscara e luvas) durante a trituração (Elke Joos, Verbeke, et al., 2016); parte da dose pode ser perdida durante a trituração, mistura e diluição do medicamento (Manessis et al., 2008); deve-se otimizar planejamento de horários de administração (Boullata \& Armenti, 2010; A. M. M. Reis et al., 2014), sendo que alguns autores sugerem que a NE seja interrompida por cerca de uma hora antes ou depois da administração da Levotiroxina (Guenter \& Boullata, 2013; A. M. M. Reis et al., 2014; A. M. M. Reis \& Cassiani, 2011).

Outras informações: absorção diminuída na insuficiência cardíaca, nas síndromes de má absorção e na diarreia (Manessis et al., 2008); trocas insuficientes das sondas e presença de resíduo alimentar e fluido gástrico no estômago provavelmente influenciam na biodisponibilidade (Manessis et al., 2008); melhor evitar fórmulas entéricas que contenham proteína de soja (Boullata \& Armenti, 2010); avaliar função tireoidiana por alguns dias/semanalmente (Boullata \& Armenti, 2010; A. M. M. Reis et al., 2014); maioria do fármaco absorvida no jejuno e íleo superior (Manessis et al., 2008).

Forma de preparo e administração: parar nutrição enteral e lavar a sonda com pelo menos $15 \mathrm{~mL}$ de água antes e depois da administração (Boullata, 2009); parte do fármaco pode precipitar-se; logo, a lavagem da sonda diminui a

Losartana (Comprimido) incidência de obstrução (Boullata, 2009).

Outras informações: produtos de degradação desconhecidos (que decaíram mais rapidamente quando expostos à luz e ao oxigênio) são encontrados após a administração via sonda (Boullata, 2009).

\begin{tabular}{|c|c|}
\hline $\begin{array}{l}\text { Metoclopramida } \\
\text { (Comprimido) }\end{array}$ & utras informações: pode causar dor abdominal, cólicas ou diarreia (Boullata \& Armenti, 2010) . \\
\hline \multirow{2}{*}{$\begin{array}{l}\text { Metronidazol } \\
\text { (Comprimido) }\end{array}$} & Forma farmacêutica: medicamento compatível com sonda entérica (Lonergan et al., 2009). \\
\hline & Outras informações: pode causar náuseas, vômitos, diarreia ou anorexia (Boullata \& Armenti, 2010). \\
\hline $\begin{array}{l}\text { Ondansentrona } \\
\text { (Comprimido) }\end{array}$ & $\begin{array}{l}\text { Outras informações: comprimidos de dissolução instantânea (sublinguais - não requerem água) podem ser opção de } \\
\text { farmacoterapia (Boullata \& Armenti, 2010). }\end{array}$ \\
\hline \multirow{3}{*}{$\begin{array}{c}\text { Paracetamol } \\
\text { (Comprimido e } \\
\text { solução oral) }\end{array}$} & $\begin{array}{l}\text { Forma farmacêutica: forma líquida disponível no mercado (Heineck et al., 2009; Kelly et al., 2011; V. G. do O. } \\
\text { Reis et al., 2010); comprimidos dispersam em água (Kelly et al., 2011). }\end{array}$ \\
\hline & $\begin{array}{l}\text { Interações potenciais: absorção significativamente prejudicada quando administrada por sonda nasogástrica em } \\
\text { pacientes com atraso do esvaziamento gástrico (Tisdale et al., 2007). }\end{array}$ \\
\hline & Outras informações: esvaziamento gástrico controla taxa de absorção (Boullata \& Armenti, 2010). \\
\hline $\begin{array}{l}\text { Polietilenoglicol } \\
\text { (Pó) }\end{array}$ & $\begin{array}{l}\text { Forma de preparo e administração: devido à viscosidade, dissolver ou diluir com grande volume de água para } \\
\text { evitar oclusão da sonda (Elke Joos, Mehuys, et al., 2016). }\end{array}$ \\
\hline $\begin{array}{l}\text { Ranitidina } \\
\text { (Xarope) }\end{array}$ & $\begin{array}{l}\text { Forma farmacêutica: forma líquida disponível no mercado (Heineck et al., 2009); sem associação com deterioração } \\
\text { ou falha da sonda (Nicole M. Phillips \& Nay, 2008). }\end{array}$ \\
\hline \multirow{3}{*}{$\begin{array}{c}\text { Sulfametoxazol } \\
+ \text { Trimetoprima } \\
\text { (Comprimido) }\end{array}$} & Forma farmacêutica: forma líquida disponível no mercado (Heineck et al., 2009). \\
\hline & Forma de preparo e administração: deve-se agitar a suspensão antes do uso (E. Joos et al., 2015). \\
\hline & Outras informações: pode causar náuseas, vômitos e anorexia (Boullata \& Armenti, 2010). \\
\hline \multirow{2}{*}{$\begin{array}{l}\text { Tansulosina } \\
\text { (Comprimido) }\end{array}$} & $\begin{array}{l}\text { Forma de preparo e administração: administrar consistentemente } 30 \text { minutos após a mesma refeição todos os dias } \\
\text { (Boullata \& Armenti, 2010). }\end{array}$ \\
\hline & es potenciais: alimentos alteram a biodisponibilidade (Boullata \& Armenti, 2010). \\
\hline
\end{tabular}




\begin{tabular}{l} 
Medicamento/ \\
$\begin{array}{c}\text { fármaco (Forma } \\
\text { farmacêutica) }\end{array}$ \\
\hline
\end{tabular}

Forma de preparo e administração: alguns autores sugerem interromper a administração de nutrição enteral por uma hora antes e uma hora depois da administração de varfarina (Boullata, 2009; Boullata \& Armenti, 2010; Dickerson, 2008; Dickerson, Garmon, Kuhl, Minard, \& Brown, 2008; Guenter \& Boullata, 2013; Krajewski \& Butterfoss, 2011; L. D. da Silva et al., 2010; L. D. Silva \& Lisboa, 2011).

Interações potenciais: uso concomitante de varfarina e nutrição enteral pode resultar na diminuição do tempo de protombina e da RNI (razão normal internacional) (DRUG-REAX®, 2018; Krajewski \& Butterfoss, 2011; A. M. M. Reis et al., 2014); biodisponibilidade do fármaco diminui quando não há interrupção da infusão da nutrição enteral (Dickerson, 2008; L. D. da Silva et al., 2010; L. D. Silva \& Lisboa, 2011); varfarina se liga a proteínas da nutrição

Varfarina (Comprimido) enteral (Dickerson, 2008; Krajewski \& Butterfoss, 2011; L. D. Silva \& Lisboa, 2011); magnésio, ferro e zinco interferem na absorção da varfarina (Boullata \& Armenti, 2010); fórmulas com alto teor de vitamina K podem antagonizar o efeito da varfarina (selecionar cuidadosamente a fórmula enteral para pacientes que recebem terapia com varfarina, evitando fórmulas com mais de $100 \mu \mathrm{g}$ de vitamina K por $1.000 \mathrm{kcal}$ ) (Boullata \& Armenti, 2010).

Outras informações: monitorar a RNI frequentemente (a cada 1-2 dias) (Boullata, 2009; Krajewski \& Butterfoss, 2011; A. M. M. Reis et al., 2014); realizar o ajuste de dose quando necessário (Krajewski \& Butterfoss, 2011; A. M. M. Reis et al., 2014); aumentos sequenciais na dose de varfarina podem ser necessários para alcançar a anticoagulação terapêutica durante terapia concomitante com nutrição enteral (Krajewski \& Butterfoss, 2011); varfarina parece ser mais absorvida no intestino delgado proximal (Boullata, 2009; Dickerson, 2008); a biodisponibilidade da varfarina após administração via sonda de jejunostomia pode ser reduzida (Boullata, 2009).

\footnotetext{
* Não encontrados dados na revisão de literatura para: amitriptilina (comprimido); bisacodil (drágea); brometo de butilescopolamina (comprimido); bromoprida (comprimido); carbonato de cálcio (comprimido); carbonato de cálcio + vitamina d (comprimido); clonazepam (comprimido); codeína + paracetamol (comprimido); dexametasona (comprimido); dexclorfeniramina (xarope); dimenidrinato (comprimido); dimeticona (solução oral); dipirona (comprimido e solução oral); enalapril (comprimido); hidroclorotiazida (comprimido); prednisolona (comprimido); prednisona (comprimido); sinvastatina (comprimido).
}

Fonte: Autores (2018).

Com base nas informações constantes na Tabela 1, foram identificados os medicamentos prescritos que apresentavam alguma limitação quanto à administração em sonda enteral, totalizando 188 medicamentos $(25,9 \%$ do total de medicamentos prescritos). Desses, 66 medicamentos $(35,1 \%)$ apresentavam, simultaneamente, interações com a NE e a forma de preparo e/ou administração diferenciada do padrão usual; outros 63 medicamentos (33,5\%) demandavam forma de preparo e/ou administração diferenciada; e os demais $59(31,4 \%)$ apresentavam apenas interação potencial com NE. Os medicamentos que mais frequentemente tiveram limitação quanto à administração em sonda enteral foram a losartana $(\mathrm{n}=29 ; 15,4 \%$ do total de limitações), atenolol ( $\mathrm{n}=28 ; 14,9 \%$ ), e captopril e amiodarona (ambos com $n=20 ; 10,6 \%$ ) (Tabela 2 ). 
Tabela 2. Frequência das limitações quanto à administração em sonda enteral identificadas entre os medicamentos prescritos de acordo com o fármaco envolvido ( $n=$ frequência de limitações). Belo Horizonte - MG. 2018.

\begin{tabular}{|c|c|c|c|c|}
\hline \multirow[b]{2}{*}{ Medicamentos (forma farmacêutica) } & \multicolumn{4}{|c|}{ Limitações quanto à administração em sonda enteral - n (\%) } \\
\hline & Interação com NE & $\begin{array}{c}\text { Forma de preparo } \\
\text { e/ou administração } \\
\text { diferenciada }\end{array}$ & $\begin{array}{l}\text { Interação com NE e forma de } \\
\text { preparo e/ou administração } \\
\text { diferenciada simultaneamente }\end{array}$ & Total \\
\hline Losartana (comprimido) & - & $29(15,4)$ & - & $29(15,4)$ \\
\hline Atenolol (comprimido) & $28(15)$ & - & - & $28(15)$ \\
\hline Captopril (comprimido) & - & - & $20(10,6)$ & $20(10,6)$ \\
\hline Amiodarona (comprimido) & $20(10,6)$ & - & - & $20(10,6)$ \\
\hline Cloreto de potássio (sol. oral e sol. injetável) & - & $17(9)$ & - & $17(9)$ \\
\hline Levotiroxina (comprimido) & - & - & $17(9)$ & $17(9)$ \\
\hline Varfarina (comprimido) & - & - & $9(4,8)$ & $9(4,8)$ \\
\hline Amoxicilina + clavulanato (pó para susp. oral) & - & $8(4,3)$ & - & $8(4,3)$ \\
\hline Ciprofloxacino (comprimido) & - & - & $8(4,3)$ & $8(4,3)$ \\
\hline Digoxina (comprimido) & - & $5(2,7)$ & $3(1,6)$ & $8(4,3)$ \\
\hline Carvedilol (comprimido) & $7(3,7)$ & - & - & $7(3,7)$ \\
\hline Lactulose (sol. oral) & - & - & $6(3,2)$ & $6(3,2)$ \\
\hline Tansulosina (comprimido) & $4(2,1)$ & - & - & $4(2,1)$ \\
\hline Polietilenoglicol (pó) & - & $3(1,6)$ & - & $3(1,6)$ \\
\hline Ácido valproico (sol. oral) & - & - & $2(1,1)$ & $2(1,1)$ \\
\hline Carbamazepina (comprimido) & - & - & $1(0,5)$ & $1(0,5)$ \\
\hline Sulfametoxazol e trimetoprima (comprimido) & - & $1(0,5)$ & - & $1(0,5)$ \\
\hline TOTAL & $59(31,4)$ & $63(33,5)$ & $66(35,1)$ & $188(100)$ \\
\hline
\end{tabular}

Legenda: NE = nutrição enteral; sol. = solução; susp. = suspensão.

Fonte: Autores (2018).

Vinte e três pacientes $(57,5 \%)$ tinham pelo menos uma prescrição com pelo menos um medicamento que apresentava alguma limitação quanto à administração via sonda enteral (Tabela 3).

Tabela 3. Número de medicamentos com limitações quanto à sua administração em sonda enteral identificadas entre os medicamentos prescritos por paciente ( $\mathrm{n}=$ número de pacientes). Belo Horizonte - MG. 2018.

\begin{tabular}{ccc}
\hline $\begin{array}{c}\text { Número de medicamentos utilizados com limitações quanto à } \\
\text { administração em sonda enteral }\end{array}$ & Frequência absoluta (n) & Frequência relativa $(\%)$ \\
\hline $\mathbf{0}$ & 17 & 42,5 \\
$\mathbf{1}$ & 5 & 12,5 \\
$\mathbf{3}$ & 3 & 7,5 \\
$\mathbf{4}$ & 1 & 2,5 \\
$\mathbf{5}$ & 2 & 5 \\
$\mathbf{6}$ & 1 & 2,5 \\
$\mathbf{7}$ & 1 & 2,5 \\
$\mathbf{8}$ & 1 & 2,5 \\
$\mathbf{1 0}$ & 2 & 5 \\
\\
$\mathbf{1 5}$ & 1 & 2,5 \\
\\
$\mathbf{1 6}$ & 2 & 5 \\
& 1 & 2,5 \\
\end{tabular}


$\mathrm{Na}$ análise multivariada, a presença de pelo menos uma limitação mostrou-se associada à idade igual ou superior a 60 anos (OR 4,67; IC95\% 1,07-20,40) (Tabela 4).

Tabela 4. Resultados das análises univariadas e multivariada das características associadas à limitação quanto à administração em sonda enteral. n=40. Belo Horizonte - MG. 2018.

\begin{tabular}{|c|c|c|c|c|c|c|}
\hline \multirow[b]{2}{*}{ Variáveis } & \multicolumn{2}{|c|}{ Interação* } & \multicolumn{2}{|c|}{ Univariadas } & \multicolumn{2}{|c|}{ Multivariada } \\
\hline & $\begin{array}{l}\text { NÃO } \\
\text { n (\%) }\end{array}$ & $\begin{array}{c}\text { SIM } \\
\text { n }(\%)\end{array}$ & OR $(\operatorname{IC} 95 \%)^{* * *}$ & p-valor $* * *$ & OR $(\mathrm{IC} 95 \%)^{\#}$ & p-valor ${ }^{\sharp}$ \\
\hline \multicolumn{7}{|l|}{ Sexo } \\
\hline Masculino & $12(46,2)$ & $14(53,8)$ & 1 & - & - & - \\
\hline Feminino & $5(35,7)$ & $9(64,3)$ & $1,54(0,40-5,88)$ & 0,384 & - & - \\
\hline \multicolumn{7}{|l|}{ Idade } \\
\hline 18 a 59 & $9(64,3)$ & $5(37,1)$ & 1 & - & 1 & - \\
\hline 60 ou mais & $8(30,8)$ & $18(69,2)$ & $4,05(1,02-16,00)$ & 0,044 & $4,67(1,07-20,40)$ & 0,040 \\
\hline \multicolumn{7}{|c|}{ Número de medicamentos } \\
\hline 1 & $12(40,0)$ & $18(60,0)$ & 1 & - & - & - \\
\hline 2 ou mais & $5(50,0)$ & $5(50,0)$ & $0,67(0,16-2,81)$ & 0,423 & - & - \\
\hline \multicolumn{7}{|c|}{ Posicionamento da sonda } \\
\hline Outros & $7(30,4)$ & $16(69,6)$ & 1 & - & 1 & - \\
\hline Estômago & $10(58,8)$ & $7(41,2)$ & $0,30(0,08-1,14)$ & 0,073 & $0,26(0,06-1,09)$ & 0,066 \\
\hline \multicolumn{7}{|c|}{ Operação de câncer de cabeça e pescoço } \\
\hline Não & $10(38,5)$ & $16(61,5)$ & 1 & - & - & - \\
\hline Sim & $7(50,0)$ & $7(50,5)$ & $0,63(0,17-2,32)$ & 0,481 & - & - \\
\hline \multicolumn{7}{|c|}{ Operação de esôfago, estômago e duodeno } \\
\hline Não & $8(44,4)$ & $10(55,6)$ & 1 & - & - & - \\
\hline $\operatorname{Sim}$ & $9(40,9)$ & $13(59,1)$ & $1,16(0,33-4,07)$ & 0,822 & - & - \\
\hline \multicolumn{7}{|c|}{ Desnutrição grave } \\
\hline Não & $10(43,5)$ & $13(53,5)$ & 1 & - & - & - \\
\hline Sim & $7(41,2)$ & $10(58,8)$ & $1,10(0,31-3,91)$ & 0,884 & - & - \\
\hline \multicolumn{7}{|l|}{ Neoplasia } \\
\hline Não & $5(31,2)$ & $11(68,8)$ & 1 & - & - & - \\
\hline Sim & $12(50,0)$ & $12(50,0)$ & $0,45(0,12-1,71)$ & 0,199 & - & - \\
\hline \multicolumn{7}{|l|}{ Hipertensão } \\
\hline Não & $13(52,0)$ & $12(48,0)$ & 1 & - & - & - \\
\hline Sim & $4(26,7)$ & $11(73,3)$ & $2,98(0,74-11,93)$ & 0,187 & - & - \\
\hline \multicolumn{7}{|l|}{ Diabetes } \\
\hline Não & $16(47,1)$ & $18(52,9)$ & 1 & - & - & - \\
\hline Sim & $1(16,7)$ & $5(83,3)$ & $4,44(0,47-42,17)$ & 0,175 & - & - \\
\hline
\end{tabular}

*Presença de pelo menos uma interação medicamento-nutrição enteral identificada ao longo do tempo de estudo

**Odds ratio (IC95\%) estimado por regressão logística

***Estimado por teste de qui-quadrado de Pearson ou exato de Fisher

\#Estimado por regressão logística stepwise

Fonte: Autores (2018).

\section{Discussão}

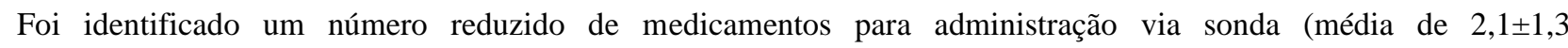
medicamentos por prescrição), mesmo tratando-se de grupo de pacientes submetidos a operações complexas e com proporção considerável apresentando desnutrição grave (42,5\% dos pacientes). Estudos com o método igual ao utilizado no presente estudo (avaliação de múltiplas prescrições durante determinado período com identificação de medicamentos prescritos via 
sonda) não foram identificados; tampouco estudos envolvendo o mesmo cenário e perfil de internação (hospital de alta complexidade, pacientes recém submetidos a operação no trato gastrointestinal e parede abdominal). Mas em um estudo realizado em um hospital do Rio de Janeiro, foi identificada uma média de 4,1 medicamentos por prescrição (M. J. S. da Silva et al., 2011). Tendo como unidade de análise pacientes, em um hospital do Ceará, foi identificada mediana de 4 medicamentos por paciente (Carvalho et al., 2010); no Rio Grande do Sul, uma média de 5 medicamentos por paciente (Heineck et al., 2009); e, em um hospital de São Paulo, uma média de 5,6 por paciente (Gorzoni, Torre Della, \& Pires, 2010).

Tal disparidade na média de medicamentos para administração via sonda pode dever-se ao fato de terem sido incluídos na análise do presente estudo apenas aqueles medicamentos que foram claramente prescritos para administração via sonda ou ostomias, uma vez que a prescrição na instituição do estudo conta com campo específico para via de administração. Entretanto, a análise baseou-se em coleta retrospectiva, não sendo possível confirmar se houve ou não alteração de via daquela prescrita originalmente no momento de administração, seja por decisão médica ou pela equipe de enfermagem, uma vez que não há registro de tais modificações na prescrição. Tal prática de mudança de via de administração sem a documentação adequada, segundo Protocolo de Segurança na Prescrição, Uso e Administração de medicamentos, caracteriza-se como erro de administração, uma vez que, durante a administração, devem ser observados, pelo menos, os cincos certos de acordo com o prescrito: medicamento certo; paciente certo; dose certa; horário certo; e via de administração certa (ANVISA, 2013; Miasso \& Cassiani, 2000).

Também foi verificada uma maioria de formas farmacêuticas sólidas prescritas para serem administradas via sonda (comprimidos e drágeas eram $72,8 \%$ dos medicamentos prescritos por essa via), assim como em estudos prévios brasileiros (60,6\% a 97,6\% dos medicamentos prescritos) (Carvalho et al., 2010; Martins et al., 2013; Renovato et al., 2010). Essas formas farmacêuticas devem passar por processo de preparo mais complexo (trituração, dispersão e solubilização), o que demanda maior atenção e tempo por parte da equipe de saúde para tais procedimentos (Oliveira, Santos, Rezende, \& Nascimento, 2020; Renovato et al., 2010). Por outro lado, sabe-se que a administração de formas líquidas via sonda também não é desprovida de desafios, devendo ser avaliada a forma adequada de preparo para prevenir a ocorrência de efeitos adversos gastrointestinais (ex.: desconforto abdominal, refluxo, náusea, vômito, diarreia), que no grupo de pacientes incluídos no presente estudo possui especial relevância, uma vez que complicações desse tipo já são esperadas após a realização de operação gastrointestinal(E. Joos et al., 2015; Klang, McLymont, \& Ng, 2013; Oliveira et al., 2020).

A administração de medicamentos via sonda está associada a maior risco de erros de medicação (Elke Joos, Mehuys, et al., 2016) e, mesmo que a forma farmacêutica mais indicada tenha sido selecionada e preparada para o paciente, a incerteza da localização da ponta distal da sonda (identificada em prontuário para apenas metade dos pacientes no presente estudo; $\mathrm{n}=20$ ) impede uma análise mais detalhada quanto a adequação da administração dos medicamentos em relação ao sítio de liberação. A ausência da documentação dessas informações relevantes para o cuidado ao paciente nos prontuários deve ser minimizada em instituições hospitalares que visam promover a segurança do paciente e fortalecer a atividade interdisciplinar voltada ao paciente sob o aporte de nutrição enteral.

Sabe-se que, dos pacientes cujo dado de posicionamento da sonda foi identificado em prontuário, a maioria ( $\mathrm{n}=17$, 85,0\%) tinha a ponta distal localizada na cavidade gástrica, sendo essa proporção superior à identificada entre pacientes avaliados em um estudo realizado em um hospital carioca (44,9\% dos pacientes com sonda de posicionamento distal gástrico) (M. J. S. da Silva et al., 2011). Opta-se por posicionar a sonda preferencialmente no estômago devido à sua maior tolerância a diversas fórmulas e medicamentos quando comparado ao intestino delgado (Miller et al., 2014; Williams, 2008). É importante destacar que parte considerável dos pacientes incluídos nesse estudo passou por operações realizadas pelo grupo de câncer de cabeça e pescoço $(n=14 ; 35,0 \%)$ e a maioria desses $(n=13)$ utilizaram a $S N G$, representando $76,5 \%$ dos pacientes que apresentavam dado de localização da ponta distal em prontuário. Geralmente, o trato gastrointestinal inferior (intestinos 
delgado e grosso) e parte do superior (esôfago e estômago) se mantinham intactos e funcionais no pós-operatório desse grupo de pacientes, o que permitia a utilização da sonda com ponta distal no estômago com segurança, e, consequentemente, reduzia limitações de administração de medicamentos irritativos para o intestino delgado ou com o processo de dissolução e absorção prejudicados em posicionamento pós-pilórico (Mcintyre \& Monk, 2014).

As limitações quanto à administração em sonda enteral mais frequentes foram relacionadas aos medicamentos com ação no sistema cardiovascular losartana, atenolol, captopril e amiodarona. A administração via sonda dos anti-hipertensivos, tem como consequência a redução significativa da biodisponibilidade no pós-operatório (atenolol), da absorção (captopril) e precipitação de parte do fármaco na sonda e formação de produtos de degradação que decaem mais rapidamente quando expostos à luz e oxigênio (losartana) (Boullata, 2009; Decloedt \& Maartens, 2009; Renovato et al., 2010). Quanto à amiodarona, é relevante apontar que, mesmo com o comprimido sendo triturável, a amiodarona adsorve em sondas feitas de policloreto de vinila e tem redução significativa da concentração sérica pós administração via sonda, podendo ser, em alguns casos, indetectável (Decloedt \& Maartens, 2009; Tisdale et al., 2007); por outro lado, sua biodisponibilidade pode aumentar mediante administração concomitante de dieta hiperlipídica (Tisdale et al., 2007). Esses fatores podem prejudicar a efetividade do tratamento, uma vez que o paciente pode apresentar elevação da pressão arterial e arritmias, que são apontados como fatores de risco para lesão renal, rehospitalização e outras complicações no período pós-operatório de pacientes que foram submetidos a operações no trato gastrointestinal, sobretudo se esses forem idosos (Hyde et al., 2018; Wu, Jiang, Ying, Han, \& Chen, 2017).

Nos estudos de Carvalho et al. (2010), de Heineck, Bueno e Heydrich (2009), e Gorzoni, Torres Della, Pires (2010), o captopril também esteve entre os medicamentos mais utilizados via sonda que possuem alguma limitação para administração por tal via; mas não foram identificados em outros estudos a losartana, atenolol ou amiodarona. Vale destacar que, no presente estudo, não foi identificada prescrição de medicamentos que não podem ser triturados via sonda. Isso é um fator positivo, que demonstra uma provável propagação de tal conhecimento entre prescritores, que, em sua maioria, são especialistas na área de cirurgia do trato gastrointestinal. Em outros estudos nacionais, foi identificada a prescrição desse grupo de medicamentos, com destaque para antiepilépticos de liberação prolongada e inibidores de bomba de prótons (E. Joos et al., 2015; Elke Joos, Mehuys, et al., 2016; Elke Joos, Verbeke, et al., 2016; L. D. Silva \& Lisboa, 2011). A administração de medicamentos de liberação modificada pode levar à liberação imediata da dose total contida na forma farmacêutica, ocasionando efeitos adversos que podem ser desde a redução ou perda de efetividade do medicamento, até danos graves e até fatais dependendo do fármaco envolvido (Elke Joos, Mehuys, et al., 2016).

A revisão permitiu verificar problemas com apenas $17(38,6 \%)$ dos 44 medicamentos prescritos para serem administrados via sonda, o que pode haver decorrido não só da possível ausência de registro de via de administração em prontuário, mas também de questões relacionadas aos estudos identificados na literatura, que foram escassos e com qualidade e métodos variados. Isso é corroborado pelo fato de não terem sido identificadas na literatura informações sobre uma parte considerável dos medicamentos (18 dos 44 medicamentos prescritos; 40,9\%), incluindo na base de dados do Micromedex ${ }^{\circledR}$, que apresentou informações para apenas 9 medicamentos (resultados não apresentados), sendo que, desses, apenas 2 (levotiroxina e varfarina) tinham informações sobre interação do medicamento especificamente com a NE; para os demais $(n=7)$, o alerta de interação era em relação à alimentos, sem maiores detalhamentos (DRUG-REAX®, 2018).

Durante a etapa de revisão, dentre os medicamentos para os quais informações foram encontradas, diferentes referências indicavam com frequência mecanismos de incompatibilidade, forma de preparo, e/ou estratégias de manejos divergentes. Como, por exemplo: no caso do ciprofloxacino, para o qual estudos divergiam nos tempos de interrupção da NE prévio à sua administração (Boullata \& Armenti, 2010; Reis et al., 2010). Informações conflitantes geram dúvidas, que, por sua vez, podem conduzir a erros. Isso reforça a necessidade de serem desenvolvidos mais estudos nessa área e também da equipe 
interprofissional se manter atualizada e levar em consideração as especificidades do paciente, avaliando caso a caso o impacto das limitações da administração de medicamentos via sonda na clínica do paciente.

Deve-se apontar que a prevalência de limitações encontrada foi elevada (57,5\% dos pacientes; $n=23)$. No grupo de pacientes incluídos no presente estudo (cirúrgicos e com média elevada de idade), isso pode ter consequências mais graves, tendo em vista a complexidade clínica e as alterações farmacocinéticas esperadas nesses quadros (Huang et al., 2015; Jansen \& Brouwers, 2012; Shi \& Klotz, 2011). Tal preocupação é reforçada pela forte associação entre idade igual ou superior a 60 anos com a presença de limitação na administração identificada na análise multivariada (OR 4,67; IC95\% 1,07-20,40). Isso indica que pacientes idosos cirúrgicos devem ser priorizados para ter sua prescrição analisada quanto à administração de medicamentos via sonda.

Nosso estudo apresentou algumas limitações como a análise retrospectiva das prescrições e a não avaliação das consequências clínicas da administração dos medicamentos via sonda. Além disso, o número reduzido de pacientes e as particularidades do cenário de estudo reduzem a aplicabilidade dos resultados identificados sobre fatores associados para outras instituições de saúde ou grupo de pacientes. Por outro lado, tais restrições são contrabalanceadas pelo fato de que, ao nosso conhecimento, trata-se do primeiro estudo a investigar a presença de limitações quanto à administração via sonda enteral de medicamentos prescritos para pacientes submetidos a operações de trato gastrointestinal e de parede abdominal, cuja fragilidade para procedimentos envolvendo tal via é relevante e deve suscitar especial atenção da equipe multiprofissional.

Ressalta-se também a importância do desenvolvimento de estudos como o aqui apresentado, uma vez que pesquisas discutindo a prevalência e fatores associados a limitações quanto à administração de medicamentos via sonda enteral, bem como desenvolvimento de banco de dados para sua identificação ainda são escassos e são importantes para o manejo adequado de pacientes sob aporte de nutrição enteral via sonda.

\section{Conclusão}

Os pacientes envolvidos no estudo apresentavam quadros clínicos complexos e parcela considerável apresentava desnutrição grave. Além disso, a associação entre idade maior ou igual a 60 anos e a identificação de pelo menos uma limitação quanto à administração via sonda enteral de medicamentos reforça a complexidade dos pacientes, o que indica a necessidade de acompanhamento mais intensificado e precaução quanto à prevenção e ocorrência de eventos adversos envolvendo a administração de medicamentos via sonda por parte da equipe multiprofissional.

$\mathrm{O}$ acesso ao compilado de informações relativas à administração de medicamentos via sonda enteral promove solidez na tomada de decisão dos profissionais da equipe multiprofissional, em especial o farmacêutico, e, consequentemente, proporciona conhecimento e um tratamento seguro e de qualidade ao paciente.

A carência numérica de estudos, baixa diversidade de medicamentos abordados nesses e variabilidade da qualidade, característica da produção científica nesse campo de conhecimento, limitou a riqueza do conteúdo disponibilizado por este trabalho. Finalmente, chama-se atenção ao ineditismo da população inserida no presente estudo e análises realizadas.

\section{Referências}

Ahn, H. S., Yook, J. H., Park, C. H., Park, Y. K., Yu, W., Lee, M. S., \& Yang, H. K. (2011). General perioperative management of gastric cancer patients at high-volume centers. Gastric Cancer, 14(2), 178-182. https://doi.org/10.1007/s10120-011-0012-x

ANVISA. (2013). Protocolo De Segurança Na Prescrição, Uso E Administração De Medicamentos. Ministério Da Saúde. Retrieved from https://www20.anvisa.gov.br/segurancadopaciente/index.php/publicacoes/item/seguranca-na-prescricao-uso-e-administracao-de-medicamentos

Boullata, J. I. (2009). Drug Administration Through an Enteral Feeding Tube. AJN, 109(10), 34-42. https://doi.org/10.1007/978-3-319-10386-0

Boullata, J. I., \& Armenti, V. T. (2010). Handbook of Drug-Nutrient Interactions. 
Bourgault, A. M., Ipe, L., Weaver, J., Swartz, S., \& J. O’Dea, P. (2007). Development of Evidence-Based Guidelines and Critical Care Nurses ' Knowledge of Enteral Feeding. American Association of Critical-Care Nurses, 27, 17-29.

Carvalho, A. M. R., Oliveira, D. C., Neto, J. E. D. H., Martins, B. C. C., Vieira, V. M. D. S. F., Silva, L. I. M. M. Da, \& Marta Maria de F. Fonteles1, $3,4$. (2010). Análise da prescrição de pacientes utilizando sonda enteral em um hospital universitário do Ceará. Revista Brasileira Farmácia Hospitalar e Serviços de Saúde, 1(1), 17-21.

Decloedt, E., \& Maartens, G. (2009). Pitfalls of administering drugs via nasogastric tubes. SAMJ Forum, 99.

Dickerson, R. N. (2008). Warfarin resistance and enteral tube feeding: A vitamin K-independent interaction. Nutrition, 24(10), 1048-1052. https://doi.org/10.1016/j.nut.2008.05.015

Dickerson, R. N., Garmon, W. M., Kuhl, D. A., Minard, G., \& Brown, R. O. (2008). Vitamin K - Independent warfarin resistance after concurrent administration of warfarin and continuous enteral nutrition. Pharmacotherapy, 28(3), 308-313. http://www.embase.com/se arch/results?subaction=viewrecord\&from=export\&id=L351328903\%5Cnhttp://dx.doi.org/10.1592/phco.28.3.308\%5Cnhttp://zp9vv3zm2k.search.serialssoluti ons.com/?sid=EMBASE\&issn=02770008\&id=doi:10.1592\%2Fphco.28.3.308\&atitle=Vitamin+K+-+Ind

DRUG-REAX®. (2018). IBM Micromedex. http://www.micromedexsolutions.com/

Gorzoni, M. L., Torre Della, A., \& Pires, S. L. (2010). Drugs and feeding tubes. Revista Da Associacao Medica Brasileira, 56(1), 17-21.

Guenter, P., \& Boullata, J. (2013). Drug administration by enteral. Nursing2013, 27-33.

Heineck, I., Bueno, D., \& Heydrich, J. (2009). Study on the use of drugs in patients with enteral feeding tubes. Pharmacy World and Science, 31(2), 145-148. https://doi.org/10.1007/s11096-008-9268-6

Heldt, T., \& Loss, S. H. (2013). Drug-nutrient interactions in the intensive care unit: Literature review and current recommendations. Revista Brasileira de Terapia Intensiva, 25(2), 162-167. https://doi.org/10.5935/0103-507X.20130028

Huang, D., Sun, Z., Huang, J., \& Shen, Z. (2015). Early enteral nutrition in combination with parenteral nutrition in elderly patients after surgery due to gastrointestinal cancer. International Journal of Clinical and Experimental Medicine, 8(8), 13937-13945.

Hyde, L. Z., Al-Mazrou, A. M., Kuritzkes, B. A., Suradkar, K., Valizadeh, N., \& Kiran, R. P. (2018). Readmissions after colorectal surgery: not all are equal. International Journal of Colorectal Disease, 33(12), 1667-1674. https://doi.org/10.1007/s00384-018-3150-3

Jack, L., Coyer, F., Courtney, M., \& Venkatesh, B. (2010). Diarrhoea risk factors in enterally tube fed critically ill patients: A retrospective audit. Intensive and Critical Care Nursing, 26(6), 327-334. https://doi.org/10.1016/j.iccn.2010.08.001

Jansen, P. A. F., \& Brouwers, J. R. B. J. (2012). Clinical Pharmacology in Old Persons. Scientifica, 2012, 1-17. https://doi.org/10.6064/2012/723678

Johnson, C. E., Cober, M. P., \& Ludwig, J. L. (2007). Stability of partial doses of omeprazole-sodium bicarbonate oral suspension. Annals of Pharmacotherapy, 41(12), 1954-1961. https://doi.org/10.1345/aph.1K246

Joos, E., Mehuys, E., Van Bocxlaer, J., Remon, J. P., Van Winckel, M., \& Boussery, K. (2015). Drug administration via enteral feeding tubes in residential care facilities for individuals with intellectual disability: An observational study. Journal of Intellectual Disability Research, 59(3), 215-225. https://doi.org/10.1111/jir.12129

Joos, Elke, Mehuys, E., Remon, J. P., Van Winckel, M., \& Boussery, K. (2016). Analysis of drug use in institutionalized individuals with intellectual disability and tube feeding. Acta Clinica Belgica: International Journal of Clinical and Laboratory Medicine, 71(2), 76-80. https://doi.org/10.1080/17843286.2015.1122332

Joos, Elke, Verbeke, S., Mehuys, E., Van Bocxlaer, J., Remon, J. P., Van Winckel, M., \& Boussery, K. (2016). Medication administration via enteral feeding tube: a survey of pharmacists' knowledge. International Journal of Clinical Pharmacy, 38(1), 10-15. https://doi.org/10.1007/s11096-015-0196-y

Jory, C., Shankar, R., Oak, K., Oates, J., \& Wilcock, M. (2017). Going down the tubes! Impact on seizure control of antiepileptic medication given via percutaneous feeding tubes. Epilepsy and Behavior, 74, 114-118. https://doi.org/10.1016/j.yebeh.2017.06.024

Kappelle, W. F. W., Siersema, P. D., Bogte, A., \& Vleggaar, F. P. (2016). Challenges in oral drug delivery in patients with esophageal dysphagia. Expert Opinion on Drug Delivery, 13(5), 645-658. https://doi.org/10.1517/17425247.2016.1142971

Kelly, J., Wright, D., \& Wood, J. (2011). Medicine administration errors in patients with dysphagia in secondary care: A multi-centre observational study. Journal of Advanced Nursing, 67(12), 2615-2627. https://doi.org/10.1111/j.1365-2648.2011.05700.x

Klang, M., McLymont, V., \& Ng, N. (2013). Osmolality, pH, and compatibility of selected oral liquid medications with an enteral nutrition product. Journal of Parenteral and Enteral Nutrition, 37(5), 689-694. https://doi.org/10.1177/0148607112471560

Kozeniecki, M., \& Fritzshall, R. (2015). Enteral Nutrition for Adults in the Hospital Setting. Nutrition in Clinical Practice, 30(5), 634-651. https://doi.org/10.1177/0884533615594012

Krajewski, K. C., \& Butterfoss, K. (2011). Achievement of therapeutic international normalized ratio following adjustment of tube feeds. Journal of Clinical Pharmacology, 51(3), 440-443. https://doi.org/10.1177/0091270010374469

Kurien, M., Penny, H., \& Sanders, D. S. (2015). Impact of direct drug delivery via gastric access devices. Expert Opinion on Drug Delivery, 12(3), 455-463. https://doi.org/10.1517/17425247.2015.966683

Lewis, S. J., Andersen, H. K., \& Thomas, S. (2009). Early enteral nutrition within 24 h of intestinal surgery versus later commencement of feeding: A systematic review and meta-analysis. Journal of Gastrointestinal Surgery, 13(3), 569-575. https://doi.org/10.1007/s11605-008-0592-x 
Lonergan, M. T., Broderick, J., Coughlan, T., Collins, D. R., \& Neill, D. O. (2009). Prescribing and enteral tubes in the general hospital. JAGS, 57(4), 736737.

Lubart, E., Berkovitch, M., Leibovitz, A., Britzi, M., Soback, S., Bukasov, Y., \& Segal, R. (2013). Pharmacokinetics of ciprofloxacin in hospitalized geriatric patients: Comparison between nasogastric tube and oral administration. Therapeutic Drug Monitoring, 35(5), 653-656. https://doi.org/10.1097/FTD.0b013e3182940426

Manessis, A., Lascher, S., Bukberg, P., Darmody, T., \& Y, V. (2008). Quantifying Amount of Adsorption of Levothyroxine by Percutaneous Endoscopic ... Source, 197-200.

Martins, M. R., Soares, A. Q., Modesto, A. C. F., Carvalho, R. F., Melo, V. V. de, \& Duarte, I. de P. (2013). Análise de medicamentos administrados por sonda em unidades de terapia intensiva em hospital de ensino. Revista Eletrônica de Enfermagem, 15(1), 191-196. https://doi.org/10.5216/ree.v15i1.15848

Mcintyre, C. M., \& Monk, H. M. (2014). Medication absorption considerations in patients with postpyloric enteral feeding tubes. American Journal of HealthSystem Pharmacy, 71(7), 549-556. https://doi.org/10.2146/ajhp130597

Miasso, A. I., \& Cassiani, S. H. (2000). Erros na administração de medicamentos: divulgação de conhecimentos e identificação do paciente como aspectos relevantes. Revista Da Escola de Enfermagem Da U S P, 34(1), 16-25.

Miller, K. R., McClave, S. A., Kiraly, L. N., Martindale, R. G., \& Benns, M. V. (2014). A tutorial on enteral access in adult patients in the hospitalized setting. Journal of Parenteral and Enteral Nutrition, 38(3), 282-295. https://doi.org/10.1177/0148607114522487

Mink, S., Muroi, C., Seule, M., Bjeljac, M., \& Keller, E. (2011). Levetiracetam compared to valproic acid: Plasma concentration levels, adverse effects and interactions in aneurysmal subarachnoid hemorrhage. Clinical Neurology and Neurosurgery, 113(8), 644-648. https://doi.org/10.1016/j.clineuro.2011.05.007

Nascimento, M. M. G. Do, \& Ribeiro, A. Q. (2010). Compilação de base de dados com recomendações para administração de medicamentos via sonda enteral. Rev. Bras. Farm. Hosp. Serv. Saúde, 1(1), 22-25.

Oliveira, R. S., Santos, S. R., Rezende, C. P., \& Nascimento, M. M. G. (2020). Recomendações para o preparo e administração de medicamentos via cateter enteral : uma revisão. Journal of Applied Pharmaceutical Sciences, (7), 88-98.

Phillips, Nicole M., \& Nay, R. (2008). A systematic review of nursing administration of medication via enteral tubes in adults. Journal of Clinical Nursing, 17(17), 2257-2265. https://doi.org/10.1111/j.1365-2702.2008.02407.x

Phillips, Nicole Margaret, \& Endacott, R. (2011). Medication administration via enteral tubes: A survey of nurses' practices. Journal of Advanced Nursing, 67(12), 2586-2592. https://doi.org/10.1111/j.1365-2648.2011.05688.x

Reis, A. M. M., Carvalho, R. E. F. L. de, Faria, L. M. P. de, Oliveira, R. C. de, Zago, K. S. de A., Cavelagna, M. F., \& Cassiani, S. H. de B. (2014). Prevalence and clinical significance of interactions drug-enteral nutrition in Intensive Care Units. Revista Brasileira de Enfermagem, 67(1), 85-90. https://doi.org/10.5935/0034-7167.20140011

Reis, A. M. M., \& Cassiani, S. H. D. B. (2011). Prevalence of potential drug interactions in patients in an intensive care unit of a university hospital in Brazil. Clinics, 66(1), 9-15. https://doi.org/10.1590/s1807-59322011000100003

Reis, V. G. do O., Candido, M. F., Jesus, R. P. de, \& Mendes-Netto, R. S. (2010). Profile of the Use of Drugs Administered Via Enteral. Revista Chil Nutr, 37(3), 293-301.

Renovato, R. D., de Carvalho, P. D., \& Rocha, R. dos S. A. (2010). Investigação da técnica de administração de medicamentos por sondas enterais em hospital geral. Revista Enfermagem UERJ, 18(2), 173-178.

Shi, S., \& Klotz, U. (2011). Age-related changes in pharmacokinetics. Current Drug Metabolism, 12(7), 601-610. https://doi.org/10.2174/138920011796504527

Silva, L. D. da, Schutz, V., Praça, B. F. M., \& Santos, M. E. R. dos. (2010). Interação fármaco-nutrição enteral: uma revisão para fundamentar o cuidado prestado pelo enfermeiro. Revista Enfermagem UERJ, 18(2), 304-310.

Silva, M. F. B. da, Brito, P. D. de, \& Guaraldo, L. (2016). Medicamentos orais de uma unidade hospitalar: adequação ao uso por cateteres enterais. Revista Brasileira de Enfermagem, 69(5), 847-854. https://doi.org/10.1590/0034-7167-2015-0081

Silva, M. J. S. da, Cava, C. E. M., Pedroso, P. K., \& Futuro, D. O. (2011). Evaluation of the profile of drug therapy administered through enteral feeding tube in a general hospital in Rio de Janeiro. Brazilian Journal of Pharmaceutical Sciences, 47(2), 331-337. https://doi.org/10.1590/S1984-82502011000200014

Silva, L. D., \& Lisboa, C. D. D. (2011). Consequências Da Interação Entre Nutrição Enteral E Fármacos Administrados Por Sondas : Uma Revisão Integrativa *. Cogitare Enferm., 16(1), 134-140.

Tisdale, J. E., Wroblewski, H. A., Hammoud, Z. T., Rieger, K. M., Young, J. V., Wall, D. S., \& Kesler, K. A. (2007). Prospective evaluation of serum amiodarone concentrations when administered via a nasogastric tube into the stomach conduit after transthoracic esophagectomy. Clinical Therapeutics, 29(10), 2226-2234. https://doi.org/10.1016/j.clinthera.2007.10.002

Wheble, G. A. C., Knight, W. R., \& Khan, O. A. (2012). Enteral vs total parenteral nutrition following major upper gastrointestinal surgery. International Journal of Surgery, 10(4), 194-197. https://doi.org/10.1016/j.ijsu.2012.02.015

Williams, N. T. (2008). Medication administration through enteral feeding tubes. American Journal of Health-System Pharmacy, 65(24), 2347-2357. https://doi.org/10.2146/ajhp080155

Wilson, N., \& Best, C. (2011). Administration of medicines via an enteral feed tube. Nursing Times, 107(41), 18-20. 
Research, Society and Development, v. 10, n. 5, e33310514927, 2021

(CC BY 4.0) | ISSN 2525-3409 | DOI: http://dx.doi.org/10.33448/rsd-v10i5.14927

http://www.embase.com/search/results?subaction=viewrecord\&from=export\&id=L366367912\%5Cnhttp://zp9vv3zm2k.search.serialssolutions.com/?sid=EMB ASE\&issn=09547762\&id=doi:\&atitle=Administration+of+medicines+via+an+enteral+feed+tube $\&$ stitle=Nurs+Times\&title=Nur

Wu, X., Jiang, Z., Ying, J., Han, Y., \& Chen, Z. (2017). Optimal blood pressure decreases acute kidney injury after gastrointestinal surgery in elderly hypertensive patients: A randomized study: Optimal blood pressure reduces acute kidney injury. Journal of Clinical Anesthesia, 43, 77-83. https://doi.org/10.1016/j.jclinane.2017.09.004

Zhao, X. F., Wu, N., Zhao, G. Q., Liu, J. F., \& Dai, Y. F. (2016). Enteral nutrition versus parenteral nutrition after major abdominal surgery in patients with gastrointestinal cancer: A systematic review and meta-analysis. Journal of Investigative Medicine, 64(5), 1061-1074. https://doi.org/10.1136/jim-2016-000083

Zhuang, C. Le, Ye, X. Z., Zhang, C. J., Dong, Q. T., Chen, B. C., \& Yu, Z. (2013). Early versus traditional postoperative oral feeding in patients undergoing elective colorectal surgery: A meta-analysis of randomized clinical trials. Digestive Surgery, 30(3), 225-232. https://doi.org/10.1159/000353136 